\title{
Self-assembled dodecyl group-modified gelatin microparticle-based hydrogels with angiogenic properties
}

\author{
Yosuke Mizuno (1) ${ }^{1,2}$ and Tetsushi Taguchi (i) ${ }^{1,2}$
}

\begin{abstract}
Supplying oxygen and nutrients to implanted cells or tissues is an important factor that improves their survivability and function in regenerative medicine. Various efforts have been made to develop angiogenic materials by incorporating and releasing growth factors such as vascular endothelial growth factor (VEGF). However, these exogenous growth factors have a short half-life under physiological conditions. We therefore designed a novel angiogenic microparticle (C12-MP) comprising Alaska pollock-derived gelatin (ApGltn) modified with a dodecyl group (C12-ApGItn) to stimulate endogenous VEGF secretion. The C12-MP suspension formed an injectable hydrogel, the rheological properties and enzymatic degradation of which were evaluated. RAW264 cells, mouse macrophage-like cells, cultured with C12-MPs, secreted significantly more VEGF than the original ApGltn MPs. Based on laser Doppler perfusion imaging, the C12-MP hydrogel clearly induced increased blood perfusion in a subcutaneous mouse model compared with the original ApGltn microparticle (Org-MP) or phosphate-buffered saline controls. Histological studies revealed that the areas of nuclear factor (NF)- $\mathrm{KB}, \mathrm{CD} 31$, and myeloperoxidase staining showed a greater increase at the site injected with C12-MPs than at the site injected with the original ApGltn microparticles or phosphate-buffered saline. The C12-MP hydrogel is a promising angiogenic material for constructing vascular beds for cell transplantation by promoting endogenous VEGF secretion without additional growth factors.
\end{abstract}

\section{Introduction}

Angiogenesis is necessary for the proliferation of implanted cells or tissues to supply oxygen and nutrients from host tissue. The incorporation of growth factors into biocompatible materials is a major strategy to induce angiogenesis before or during the transplantation of cells. Tabata et al. ${ }^{1}$ demonstrated vascular endothelial growth factor (VEGF) incorporated into a collagen hydrogel that showed a controlled release profile of VEGF and angiogenesis in vivo. Peattie et al. $^{2}$ reported a hyaluronic acid hydrogel crosslinked with poly(ethylene glycol) incorporated with VEGF and keratinocyte growth factor (KGF).

\footnotetext{
Correspondence: Tetsushi Taguchi (TAGUCHI.Tetsushi@nims.go.jp)

${ }^{1}$ Graduate School of Science and Technology, Degree Programs in Pure and Applied Sciences, University of Tsukuba, Tsukuba, Japan

${ }^{2}$ Polymers and Biomaterials Field, Research Center for Functional Materials,

National Institute for Materials Science, Tsukuba, Japan
}

This hydrogel induced microvascular formation around the implant site by the released VEGF and KGF. Although these growth factors effectively promote angiogenesis, their instability under physiological conditions is considered a limitation to their practical application ${ }^{3}$ because VEGF and fibroblast growth factor-2 (FGF-2) have half-lives of only 50 and $9.6 \mathrm{~min}$, respectively ${ }^{4,5}$. To overcome these problems, we focused on the use of endogenous growth factors to achieve angiogenesis. Proangiogenic growth factors such as VEGF are mainly secreted from macrophages in response to ischemia, injury, and inflammation ${ }^{6}$.

Saturated fatty acids (SFAs), such as capric acid (C10), lauric acid (C12), and palmitic acid (C16), behave like lipopolysaccharide (LPS), which is a component of the outer membrane of Gram-negative bacteria $^{7-9}$. SFAs induce toll-like receptor 4 (TLR4)-mediated inflammation and enhance the secretion of proinflammatory cytokines 

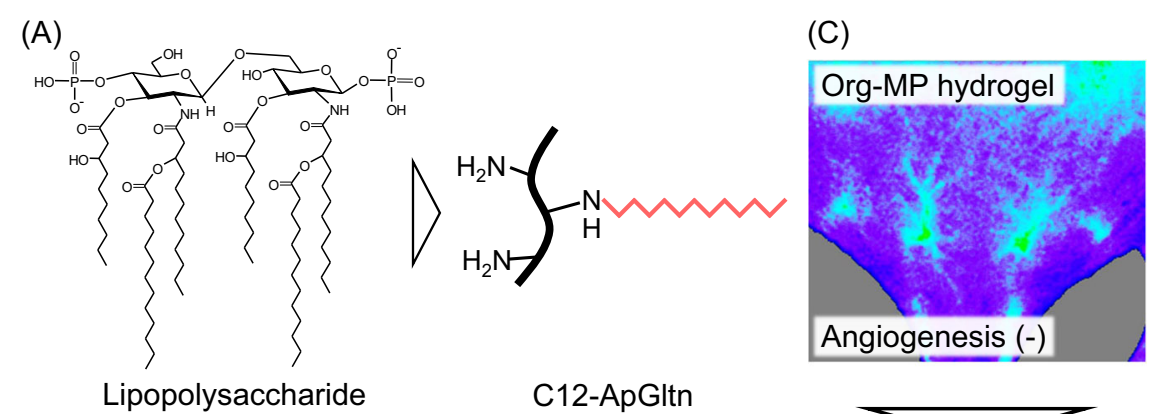

(B)
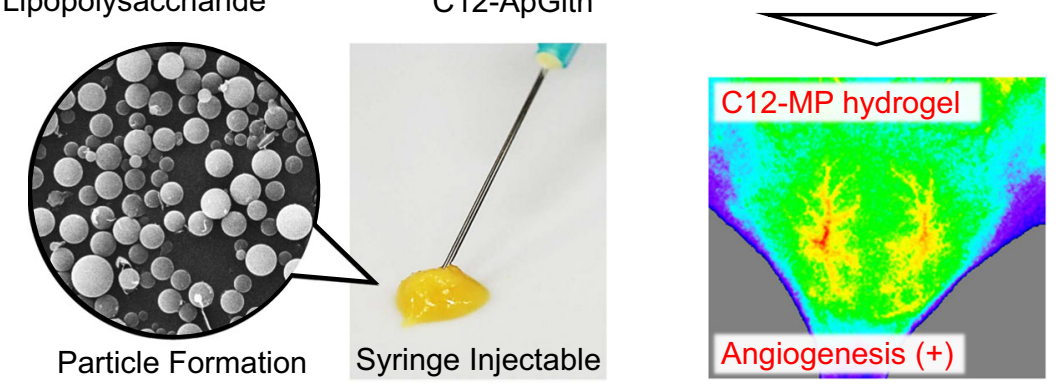

Fig. 1 Overview of the angiogenic C12-MP hydrogel. a The LPS-mimicking structure of C12-ApGltn. $\mathbf{b}$ The aqueous suspension of C12-MP had shear-thinning properties and was injectable through a needle. c The increased blood flow in the C12-MP hydrogel-injected site.

such as tumor necrosis factor (TNF)- $\alpha^{10-15}$. We previously reported that a porous gelatin sponge comprising hydrophobically modified gelatin (hm-Gltn) induced angiogenesis in vivo without incorporating growth factors ${ }^{16}$. We also reported that a dodecyl group-modified (C12) Alaska pollock-derived gelatin (ApGltn) (C12-ApGltn), which mimics an LPS of Pseudomonas aeruginosa (Fig. 1a), formed a self-assembled hydrogel that stimulated VEGF secretion in vitro and in vivo through TLR4-mediated pathways ${ }^{17}$. Although the C12-ApGltn self-assembled hydrogel stimulated angiogenesis in vivo by itself without additional growth factors, the resulting hydrogel did not achieve sustained angiogenesis because of its fast degradation and dispersion in vivo. Furthermore, we recently reported that micrometer-sized particles based on hydrophobically modified ApGltn can be obtained using a coacervation method ${ }^{18}$. The resulting particle formed an enzymatically stable, self-assembled particle-based hydrogel when in contact with water.

In the present study, C12-ApGltn was made into microparticles followed by thermal crosslinking to enhance degradation resistance for sustained angiogenesis. We characterized the hydrophobicity, enzymatic degradation rate, and rheological properties of the resulting particle-based hydrogels followed by in vivo angiogenesis evaluations (Fig. 1c).

\section{Materials and methods Materials}

ApGltn (molecular weight $=33,000$ ) was purchased from Nitta Gelatin (Osaka, Japan). Dodecanal was purchased from Tokyo Chemical Industry (Tokyo, Japan). 2-Picoline borane (pic- $\mathrm{BH}_{3}$ ) was purchased from Junsei Chemical (Tokyo, Japan). Collagenase, water-soluble tetrazolium (WST)-8 reagent (Cell Count Reagent SF) and Dulbecco's phosphate-buffered saline (PBS) were obtained from Nacalai Tesque (Kyoto, Japan). 2,4,6-Trinitrobenzenesulfonic acid (TNBS), triethylamine, 2-aminoethanol, ethanol, dimethyl sulfoxide (DMSO), and 10\% formalin neutral buffer solution were purchased from Wako Pure Chemical Industries (Osaka, Japan). Minimum essential media (MEM)- $\alpha$, MEM nonessential amino acids (NEAAs) and penicillin-streptomycin (Pen Strep) were purchased from Thermo Fischer Scientific (MA, USA). Fetal bovine serum (FBS) was purchased from Sigma-Aldrich (MO, USA). The TLR4 peptide inhibitor set (VIPER) was purchased from Novus Biologicals (CO, USA). Ultrapure LPS-EB was purchased from InvivoGen (CA, USA).

\section{Synthesis and characterization of C12-ApGltn}

Quantification of the amino groups in ApGltn was initially conducted using the TNBS method as previously reported $^{19-22}$. Briefly, $100 \mu \mathrm{l}$ of ApGltn dissolved in a 50\% $\mathrm{DMSO} / \mathrm{H}_{2} \mathrm{O}$ solution at $0.5 \mathrm{w} / \mathrm{v} \%$ was dispensed into a 48 well plate followed by the addition of TNBS and triethylamine dissolved in $1 \% \mathrm{DMSO} / \mathrm{H}_{2} \mathrm{O}$. After shaking for $1 \mathrm{~min}$, the microplate was incubated for $2 \mathrm{~h}$ at $37^{\circ} \mathrm{C}$. The absorbance at $335 \mathrm{~nm}$ was measured with a microplate reader (Spark 10 M; Tecan, Männedorf, Switzerland), and the number of amino groups was calculated using a calibration curve generated by 2-aminoethanol instead of ApGltn. 


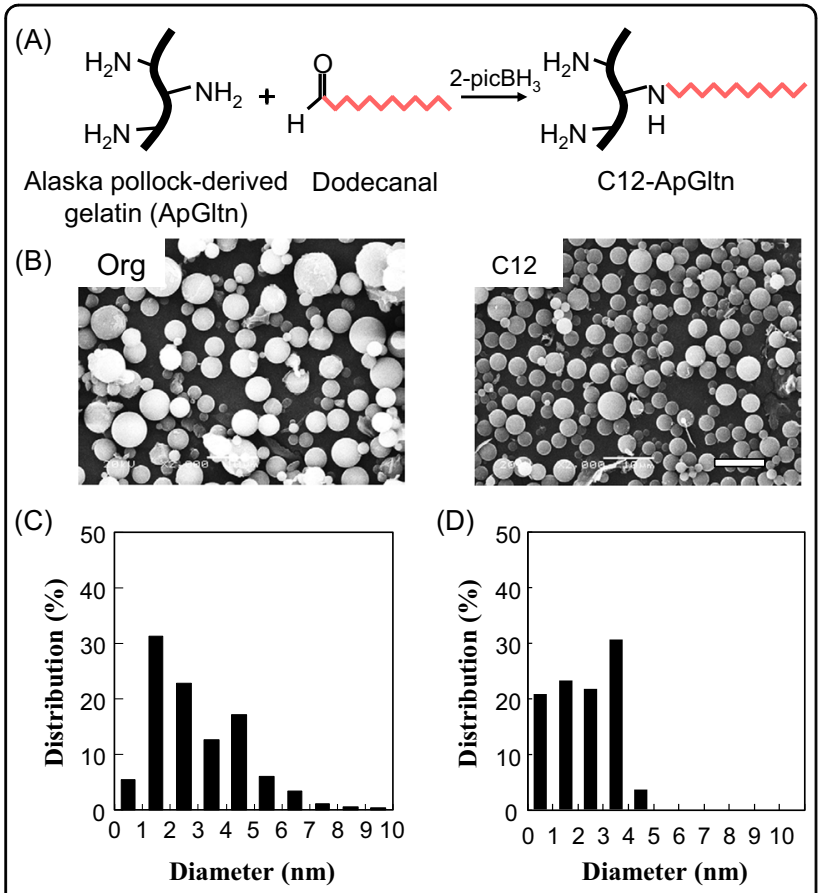

Fig. 2 The fabrication of C12-ApGltn and MPs. a Synthesis of C12ApGltn. b SEM images of the Org- and C12-MPs (scale bar $=10 \mu \mathrm{m}$ ) and particle diameters of the $\mathbf{c}$ Org-MPs and $\mathbf{d}$ C12-MPs calculated by ImageJ software

ApGltn was modified with a dodecyl group by reductive amination ${ }^{23-26}$ as previously described ${ }^{22,27}$ (Fig. 2a). Briefly, ApGltn was dissolved in $30 \% \mathrm{EtOH} / \mathrm{H}_{2} \mathrm{O}$ at $20 \mathrm{w} /$ $\mathrm{v} \%$, and 1.5 eq of dodecanal on the remaining ApGltn amino groups dissolved in ethanol was added. After an 18 - $\mathrm{h}$ reaction, the solution was added dropwise to 10 times the volume of cold ethanol to precipitate C12ApGltn. The precipitate was filtered under vacuum and washed three times with $\mathrm{EtOH}$ to remove unreacted

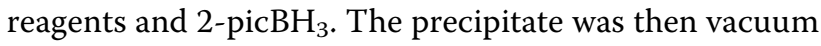
dried at room temperature $\left(25^{\circ} \mathrm{C}\right)$ to obtain C12-ApGltn as a white powder and subsequently weighed. Nonmodified ApGltn (Org-ApGltn) was similarly synthesized but without the addition of dodecanal or 2- $\mathrm{picBH}_{3}$. The modification ratio of C12-ApGltn was quantified using the TNBS method by comparing the absorbance at $335 \mathrm{~nm}$ with that of Org-ApGltn. Modification of the hydrophobic groups was confirmed by Fourier transform infrared spectroscopy (FT-IR) (ALPHA II; Bruker Corp., Billerica, MA, USA) and ${ }^{1} \mathrm{H}$ nuclear magnetic resonance $\left({ }^{1} \mathrm{H}-\mathrm{NMR}, \quad J N M-A L 300 ;\right.$ JEOL, Tokyo, Japan). The modification ratio of the dodecyl group was also calculated by comparing the Org- and C12-ApGltn spectra. The arginine-based peak at 2.95-3.25 ppm was normalized in each spectrum, followed by comparing the peak integration of the $\varepsilon$-methylene protons of lysine $(2.70-2.90 \mathrm{ppm})$.

\section{Preparation of the ApGltn microparticles}

Although the aqueous solution of C12-ApGltn became a hydrogel due to the hydrophobic crosslinking between dodecyl groups, the hydrogel was easily dispersed into water. Therefore, C12-ApGltn was transformed into microparticles by coacervation followed by thermal crosslinking to improve water resistance ${ }^{28}$. Briefly, Organd C12-ApGltn were dissolved in distilled water and a $20 \% \mathrm{EtOH} / \mathrm{H}_{2} \mathrm{O}$ solution at $5 \mathrm{w} / \mathrm{v} \%$, respectively. EtOH was added to each solution with vigorous stirring to dilute each solution twofold and precipitate the microparticles. The solution with microparticles was cooled at $-20^{\circ} \mathrm{C}$ for $18 \mathrm{~h}$ followed by lyophilization. The resulting Org- and C12-microparticles (MPs) were subjected to mortar pulverization and sieving using a $32-\mu \mathrm{m}$ opening mesh. Org- and C12-MPs were then placed on a holder with carbon tape for scanning electron microscopy (SEM). (S-4800 ultrahigh-resolution SEM, HITACHI, Japan). Platinum was sputtered on the MPs at a thickness of $10 \mathrm{~nm}$. The particle size was quantified from nine SEM images using ImageJ software. The polydispersity index (PDI) of the MPs was calculated by the following equation $^{29,30}$

$$
\operatorname{PDI}=\left(\frac{\sigma}{d}\right),
$$

where $\sigma$ and $d$ indicate the standard deviation and average diameter of the MPs, respectively. The obtained MPs were then thermally crosslinked under vacuum at $150{ }^{\circ} \mathrm{C}$ for 3,6 , and $9 \mathrm{~h}$ to introduce MPs with degradation resistance (MP3, MP6, and MP9, respectively). The remaining amino group of the MPs after thermal crosslinking was determined by the TNBS method with slight changes. Briefly, MP3, MP6, and MP9 were dissolved in a $50 \% \mathrm{DMSO} / \mathrm{H}_{2} \mathrm{O}$ solution at $0.5 \mathrm{w} / \mathrm{v} \%$ followed by incubation for $2 \mathrm{~h}$ at $50^{\circ} \mathrm{C}$. Next, $100 \mu \mathrm{l}$ of solution was transferred to a 48 -well plate, and $100 \mu \mathrm{l}$ of $1 \%$ TNBS solution and $1 \%$ TEA solution were added to each well. After $2 \mathrm{~h}$ of incubation at $37^{\circ} \mathrm{C}$, the reaction was stopped by the addition of $50 \mu \mathrm{l}$ of $6 \mathrm{~N} \mathrm{HCl}$. The absorbance at $335 \mathrm{~nm}$ was measured for each well to determine the remaining number of primary amino groups in the Organd C12-MPs.

\section{Surface contact angle of the particles}

To determine the hydrophobicity of thermally crosslinked Org- and C12-MPs, the surface contact angle was measured by the sessile-drop method ${ }^{27}$. Briefly, doublesided tape was pasted on the slide glass and then covered with Org- and C12-MPs. The contact angle measurements started $0.5 \mathrm{~s}$ after a $3 \mu \mathrm{l}$ PBS droplet was dropped on the MP surface using DropMaster DM-701 (Kyowa Interface Science, Japan) for $15 \mathrm{~s}$. 


\section{Rheological properties}

Org- and C12-MPs were dispersed in PBS at $40 \mathrm{w} / \mathrm{v} \%$ to form a particle suspension. These suspensions were placed on the stage of an MCR 301 rheometer (Anton Paar GmbH, Graz, Austria) sandwiched between flat PP10 plates separated by a gap of $1.0 \mathrm{~mm}$. A strain sweep was initially carried out to determine the linear viscoelastic region (LVE) for subsequent measurements at a frequency of $1 \mathrm{rad} / \mathrm{s}$ and temperature of $37^{\circ} \mathrm{C}^{31}$. Frequency sweeps were performed from 0.1 to $100 \mathrm{rad} / \mathrm{s}$ at $1 \%$ strain and $37^{\circ} \mathrm{C}$ to determine the storage modulus $\left(G^{\prime}\right)$ and loss modulus $\left(G^{\prime \prime}\right)$. To compare the elasticity of Org- and C12ApGltn, the $\mathrm{G}^{\prime}$ of the frequency sweep at $1 \mathrm{rad} / \mathrm{s}$ was taken as the elastic modulus ${ }^{32}$. Moreover, the effect of the shear rate on the viscosity was also measured to evaluate the behavior of the MP suspension during syringe injection. The same samples used in the strain and frequency sweep were employed to measure the viscosity with a changing shear rate from 0.01 to $1 \mathrm{~Hz}$ at $37^{\circ} \mathrm{C}$.

\section{Degradation test}

The enzymatic degradation rates of the Org- and $\mathrm{C} 12$ MPs were evaluated using collagenase. In brief, $80 \mathrm{mg}$ of C12-ApGltn was dissolved in $200 \mu \mathrm{l}$ of PBS to prepare the hydrogel in 2.5-ml tubes followed by the addition of PBS to the tube to allow the hydrogel to swell for $2 \mathrm{~h}$. After centrifuging the tubes to precipitate the particles, the supernatant was removed, and $500 \mu \mathrm{l}$ of collagenase solution $(10 \mathrm{U} / \mathrm{ml}$ in $\mathrm{PBS})$ was added. After up to $1080 \mathrm{~min}$ of incubation at $37^{\circ} \mathrm{C}$, the tubes were centrifuged to separate the hydrogel from the supernatant, and the supernatant was discarded. The weight of the C12-MP hydrogel was compared with the initial weight to determine the remaining \% hydrogel volume.

\section{In vitro cell response}

Mouse macrophage-like RAW264 cells were cultured in MEM $\alpha$ supplemented with $10 \%$ FBS, $1 \%$ NEAAs, and $1 \%$ Pen Strep. Cells were then seeded on a 96-well plate at $2.0 \times 10^{4}$ cells/well and incubated for $18 \mathrm{~h}$ at $37^{\circ} \mathrm{C}$ and $5 \%$ $\mathrm{CO}_{2}$. Org- and C12-MPs dispersed in PBS at 0.5, 1 and $2 \mathrm{w} / \mathrm{v} \%$ were sterilized by ultraviolet (UV) irradiation for $1 \mathrm{~h}$. To confirm the TLR4-mediated pathway, VIPER, a TLR4 blocking peptide, was added at $30 \mathrm{mM}$ to the culture medium followed by incubation for $1 h^{33}$. Org- and C12-MP suspensions were then added to the culture plate at a 10 -fold dilution to obtain final concentrations of 0.05 , 0.1 , and $0.2 \mathrm{w} / \mathrm{v} \%$. RAW264 cells were also cultured in media supplemented with and without $10 \mathrm{ng} / \mathrm{ml} \mathrm{LPS} \mathrm{as} \mathrm{a}$ positive and negative control, respectively. After incubation for $24 \mathrm{~h}$, the supernatants were separated for the enzyme-linked immunosorbent assay (ELISA) followed by evaluation of the cell viability using a WST-8 reagent according to the manufacturer's instructions. The amounts of secreted VEGF and TNF- $\alpha$ were quantified with an ELISA kit (Quantikine ELISA kit; R\&D Systems, Minneapolis, MN, USA) according to the manufacturer's instructions.

\section{Subcutaneous implantation of the C12-MP and laser Doppler perfusion imaging (LDPI) evaluation}

Animal experiments were approved by the Animal Care and Use Committee of National Institute for Material Science (NIMS), Japan, and performed in accordance with NIMS Regulations Pertaining to Animal Testing. Hos:HR1 hairless mice (4- week old; Hoshino Laboratory Animals, Ibaraki, Japan) were used to evaluate the tissue response of the Org- and C12-MP hydrogels. In brief, Org- and C12-MPs were dispersed in PBS at $40 \mathrm{w} / \mathrm{v} \%$ followed by UV sterilization for $1 \mathrm{~h}$. Next, $50 \mu \mathrm{l}$ of each sample was injected through a 21 -gauge needle into the backs of the mice exposed to inhalational anesthetics. LDPI measurements were performed immediately after implantation and for up to 22 days using an OZ-2Pro (OMEGAWAVE, Tokyo, Japan). To stabilize the blood flow in different mice, the respiratory rate was set to once per second. For the histological studies, PBS, Org-MP3, and C12-MP3 hydrogels were subcutaneously injected into mice, and after 2 days, the mice were sacrificed by intraperitoneal injection of a lethal dose of somnopentyl. The skin tissue around the injection site was dissected and fixed with a $10 \%$ formalin neutral buffer solution. The fixed tissues were then embedded in paraffin and cut into sections followed by staining with hematoxylin and eosin (H\&E) or labeling with antibodies against CD31, NF-KB, and myeloperoxidase (MPO) (Abcam, Cambridge, UK). The labeled areas of the tissue sections were quantified using ImageJ software.

\section{Statistical analysis}

Data are shown as the mean \pm standard deviation and were analyzed with Student's $t$ test. Differences were considered statistically significant at $p<0.05$. One-way analysis of variance (ANOVA) followed by the Tukey-Kramer method was used to determine whether there were any statistically significant differences between the means of three or more independent groups.

\section{Results and discussion \\ Synthesis of C12-ApGltn}

The yields of the obtained Org- and C12-ApGltn were 90.2 and $81.6 \%$, respectively. The modification ratio of the dodecyl group in C12-ApGltn was $32.6 \mathrm{~mol} \%$ for the total amount of amino residues in ApGltn (Table 1). The modification ratio of the dodecyl group in C12-ApGltn was also calculated by NMR spectroscopy (Fig. S1A). The integration of the Arg peak at 2.95-3.25 ppm was normalized to 1.0 (Fig. S1C) $^{34,35}$. The peak integrations of the 
Table 1 Characterizations of Org- and C12-MPs.

\begin{tabular}{lccccc}
\hline Abbreviation & Modification ratio $(\mathbf{m o l} \%)$ & Average diameter $(\boldsymbol{\mu m})$ & PDI $(-)$ & Crosslinking time $(\mathbf{h})$ & Half-life in collagenase solution $(\mathbf{h})$ \\
\hline Org-MP & - & 2.66 & 0.34 & - & - \\
- MP3 & - & - & - & 3 & 7.18 \\
-MP6 & - & - & - & 6 & 9.47 \\
-MP9 & - & - & - & 9 & 9.07 \\
C12-MP & 32.6 & 2.22 & 0.27 & - & - \\
- -MP3 & - & - & - & 3 & 19.58 \\
- MP6 & - & - & - & 6 & 21.82 \\
- MP9 & - & - & - & 9 & 20.86 \\
\hline
\end{tabular}

The modification ratio of the dodecyl group in C12-ApGltn was measured before transformation into C12-MPs. The average diameter and PDI were calculated from the SEM images. The half-lives of the Org- and C12-MP hydrogels under physiological conditions were calculated from the degradation profile (Fig. 4f).

$\varepsilon$-methylene protons of lysine at $2.70-2.90 \mathrm{ppm}$ in Organd C12-ApGltn were 0.562 and 0.435 , respectively, indicating a $29 \%$ decrease in the $\varepsilon$-methylene of lysine by dodecyl modification (Fig. S1B, C). Therefore, quantitation of the modification ratio from the NMR spectra was correlated with that from the TNBS method.

\section{Preparation and characterization of Org- and C12-MPs}

SEM images of Org- and C12-MP showed that these particles were successfully formed with a spherical shape using the coacervation method, and almost no deformed particles were observed (Fig. 2b). The particle diameter distribution was calculated from SEM images using ImageJ software (Fig. 2c, d). Most of the diameters of the MPs were distributed within 1-4 $\mu \mathrm{m}$; however, the number of C12-MPs with diameters $<1 \mu \mathrm{m}$ was greater than that of the Org-MPs. Moreover, there were almost no C12-MPs $>5 \mu \mathrm{m}$, whereas $\sim 10 \%$ of the Org-MPs exceeded $5 \mu \mathrm{m}$ in diameter. These results correlated with the higher PDI of Org-MP than C12-MP (Table 1). Additionally, we observed hydrophobic moieties on the surface of the C12MPs using FT-IR. Figure S2 shows the FT-IR spectra of the Org- and C12-MPs. The absorbances at 2853 and $2927 \mathrm{~cm}^{-1}$, which correlate to $\mathrm{C}-\mathrm{H}$ stretching, were increased in the spectrum of C12-MP, which indicates that the dodecyl group existed on the surface of C12-MP. The remaining amino groups of Org- and C12-MPs with different thermal crosslinking times were evaluated using the TNBS method. Figure S3 shows the relative ratio of the remaining amino groups in the Org- and C12-MPs with thermal crosslinking compared with that in Org- and C12-ApGltn. The remaining amino groups significantly decreased with increasing thermal crosslinking time, indicating successful covalent crosslinking. However, there was no significant difference between Org- and C12-MP crosslinked for 6 and $9 \mathrm{~h}$ due to fewer amino groups in C12-ApGltn because of $\sim 33 \%$ modification.

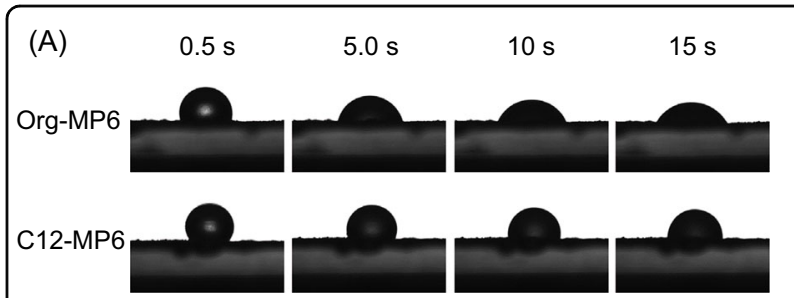

(B)

(C)

$\begin{array}{lll}\bullet \text { Org } 3 h & \circ \text { Org } 6 h & \circ \text { Org } 9 h \\ \Delta \mathrm{C} 123 \mathrm{~h} & \Delta \mathrm{C} 126 \mathrm{~h} & \Delta \mathrm{C} 129 \mathrm{~h}\end{array}$
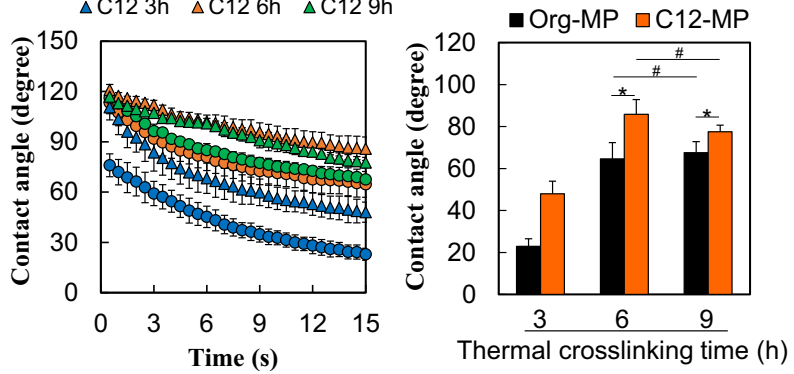

Fig. 3 Surface contact angle measurements of the Org- and C12MPs. a Representative images of the behavior of PBS droplets on the Org-MP6 and C12-MP6 surfaces for $15 \mathrm{~s}$. b Surface contact angle measurements over $15 \mathrm{~s}(n=3)$. c Comparison of the contact angle on Org- and C12-MPs at $15 s .{ }^{*} p<0.5,{ }^{\#} p>0.05$.

Therefore, only a few amino groups in C12-ApGltn could contribute to the formation of an amide bond with a carboxylic acid during thermal crosslinking.

\section{Contact angle measurements}

The contact angles of the Org- and C12-MPs were measured to evaluate the bulk hydrophobicity of the MPs. Figure 3a shows the PBS droplets on the Org-MP6 and C12-MP6 layers. The PBS droplet was more quickly absorbed onto Org-MP6 than the C12-MP6 layer. The time course of the surface contact angle on MP3, MP6, and MP9 indicated a significantly faster decrease of the 
contact angle on Org- and C12-MP3, whereas Org-MP6 and Org-MP9, as well as C12-MP6 and C12-MP9, showed similar behavior in the decrease of the contact angle (Fig. 3b). Figure 3c compares the contact angles on the MP layers at $15 \mathrm{~s}$ and indicates that only Org- and C12MP3 had significantly lower contact angles than Org- and C12-MP6 and Org- and C12-MP9. Although modification with the dodecyl group contributed to the significant difference in the surface contact angle, there was no significant difference between the MP6 and MP9 layers. The longer thermal crosslinking significantly affected the crosslinking ratio (Fig. S3), but that difference was not considered dominant regarding the surface contact angle and water absorption. On the other hand, from a topological perspective, the C12-MP layer was considered to be smoother than the Org-MP layer because the C12-MPs contained a larger number of smaller particles than the Org-MPs. Therefore, the increased contact angle of the C12-MPs was attributed to not only the increased hydrophobicity but also the decreased surface roughness of the C12-MP layers.

\section{Viscoelastic properties of the C12-MPs}

Before measuring the frequency sweep, the LVE was determined by the strain sweep (Fig. S4A, B). The LVE indicates the strain range in which the measurement can be executed without breaking the samples. Therefore, the strain was set at $1 \%$ to measure the frequency sweep. The frequency sweep measurement results showed that the storage modulus $\left(\mathrm{G}^{\prime}\right)$ of the Org- and C12-MP suspensions exceeded their loss modulus $\left(G^{\prime \prime}\right)$ within the frequency range (Fig. 4a, b), which indicates that all concentrations of the Org- and C12-MP suspensions formed a hydrogel. Thermal crosslinking promoted dehydration condensation between the carboxylic group and amino group in ApGltn, preventing its decomposition in water. Moreover, Org- and C12-MP absorbed water and swelled into a microgel-like condition, which formed the hydrogel as a whole. Alternatively, the effects of the change in the crosslinking time were also evaluated. The $\mathrm{G}^{\prime}$ of the hydrogels was dependent on the hydrodynamic radius of the swollen MPs. A longer crosslinking time led to increase amide bonding formation and crosslinking density of the surface of MPs, resulting in a lower swelling ratio and smaller hydrodynamic radius. Therefore, Organd C12-MP3, which have lower crosslinking densities, have larger hydrodynamic radii and sparser distributions in the hydrogel, which resulted in lower $\mathrm{G}^{\prime}$ values among all hydrogels (Fig. 4c). Conversely, MP6 and MP9 have a greater $\mathrm{G}^{\prime}$ than Org- and C12-MP3 because of the greater crosslinking density and smaller hydrodynamic radii, which leads to the formation of a dense hydrogel (Fig. 4c). However, no significant increase was confirmed between the $G^{\prime}$ of the Org-MP6 and Org-MP9 hydrogels, indicating that the difference in the crosslinking ratio between Org-MP6 and Org-MP9 did not significantly affect their $\mathrm{G}^{\prime}$ values. By comparing the Org- and C12-MP hydrogels, the $\mathrm{G}^{\prime}$ of the C12-MP hydrogels was significantly higher than that of Org-MP hydrogels among all crosslinking times (Fig. 4c). These results were due to the increased interaction between C12-MPs caused by the dodecyl group modification in C12-ApGltn. On the other hand, we also evaluated $\tan \delta$, which is the ratio of $\mathrm{G}^{\prime \prime}$ to $\mathrm{G}^{\prime}$, to evaluate the viscous or elastic features of the MP hydrogels. Figure $4 \mathrm{~d}$ shows that the significantly higher $\tan \delta$ values of the Org- and C12-MP3 hydrogels were confirmed. These results indicated that the Org- and C12MP3 hydrogels dissipated the loaded oscillation stress and behaved like viscous materials due to the large hydrodynamic radius and sparse distribution in hydrogels. Organd C12-MP6 as well as Org- and C12-MP9 hydrogels had lower $\tan \delta$ values compared with the respective MP3 hydrogels, indicating that they formed packed structures, presumably due to their smaller hydrodynamic diameters, and behaved like elastic materials. However, the $\tan \delta$ values of C12-MP6 and C12-MP9 were significantly higher than those of Org-MP6 and Org-MP9, respectively. Hydrated Org-MPs tend to interact with other MPs, and the hydrogel as a whole behaves like a chemically crosslinked hydrogel. However, C12-MPs had a lower tendency of hydration because of their higher hydrophobicity and formed a denser packing structure compared with OrgMPs, resulting in the dissipation of loaded force by deforming the packing structure of C12-MPs.

Alternatively, the shear-thinning properties of the Organd C12-MP hydrogels were evaluated using a rotational rheometer. Figure $4 \mathrm{e}$ indicates that the viscosity of the Org- and C12-MP hydrogels decreased with increasing shear rate, indicating that those hydrogels could be injected via syringe. Org- and C12-MP hydrogels were assumed to form aggregates in particle suspensions by hydrophobic interactions between particles. When the shear stress was loaded onto the hydrogels, those interactions could not tolerate the shear stress, and the particle aggregation eventually deformed ${ }^{36}$. Therefore, the Organd C12-MP hydrogels had shear-thinning behavior and were injectable through a 21-gauge needle (Fig. 1b).

\section{Degradation profile of the Org- and C12-MP hydrogels}

To evaluate the effects of the hydrophobic group in C12-ApGltn and the crosslinking time on the degradation rate, MP hydrogels were immersed in a collagenase solution for up to $48 \mathrm{~h}$. The degradation rate of the C12MP hydrogels was slower than that of the Org-MP hydrogels across all crosslinking times (Fig. 4f). This result was likely due to the greater interparticle interactions of the C12-MP hydrogels than that of the Org-MP hydrogels because of the additional physical crosslinking by the 

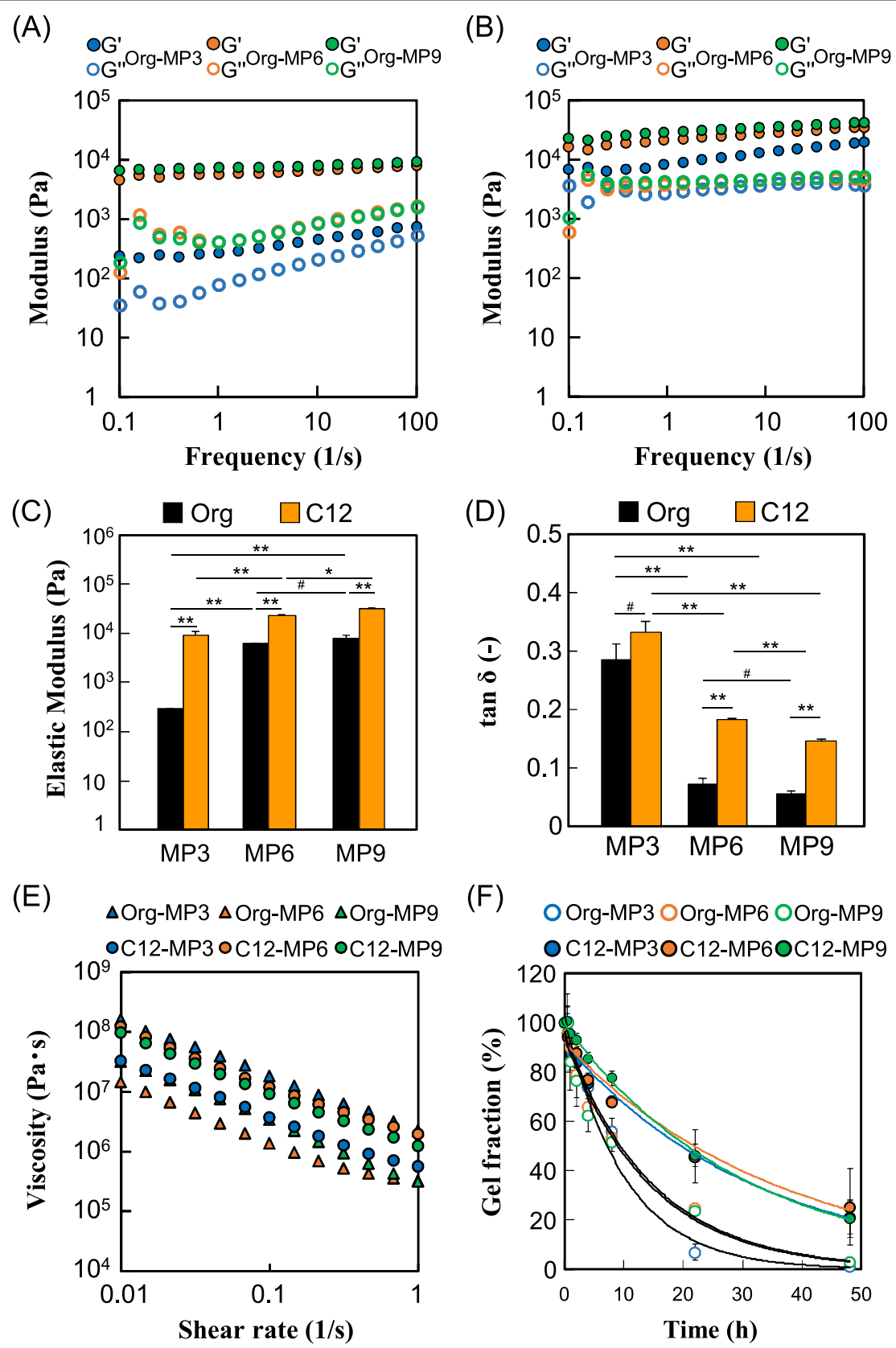

Fig. 4 Physicochemical propertes of MP hydrogels. Storage $\left(G^{\prime}\right)$ and loss $\left(G^{\prime \prime}\right)$ moduli of the (a) Org- and (b) C12-MP hydrogels with 3-9h of thermal crosslinking measured by frequency sweep rheometry. $\mathbf{c}$, $\mathbf{d}$ Comparison of the elastic modulus and $\tan \delta$ at $1 \mathrm{~Hz}$ taken from the rheometry of the Org- and C12-MP hydrogels. e Shear-thinning properties of the MP hydrogels. $\mathbf{f}$ Enzymatic degradation profile of the Org-and C12-MP hydrogels with different crosslinking times.

dodecyl group in C12-ApGltn that suppressed penetration of the collagenase solution into the C12-MP hydrogel matrix. Alternatively, the gel fraction of the Org-MP3 hydrogel remained at only $7 \%$ after incubation for $24 \mathrm{~h}$, whereas that of the Org-MP6 and Org-MP9 hydrogels was $>20 \%$. Additionally, the gel fraction of the C12-MP9 hydrogel was greater than that of the C12-MP3 and C12-MP6 hydrogels by $8 \mathrm{~h}$; however, no difference was confirmed among the C12-MP3, C12-MP6, and C12-MP9 hydrogels after $24 \mathrm{~h}$. These results indicate that a longer crosslinking time tends to enhance the degradation resistance because of the increased covalent crosslinking density. The degradation rate of the C12-MP hydrogels was, however, affected by hydrophobic modification and crosslinking time. The half-life of Org- and C12-MP hydrogels was also calculated by using the approximate 


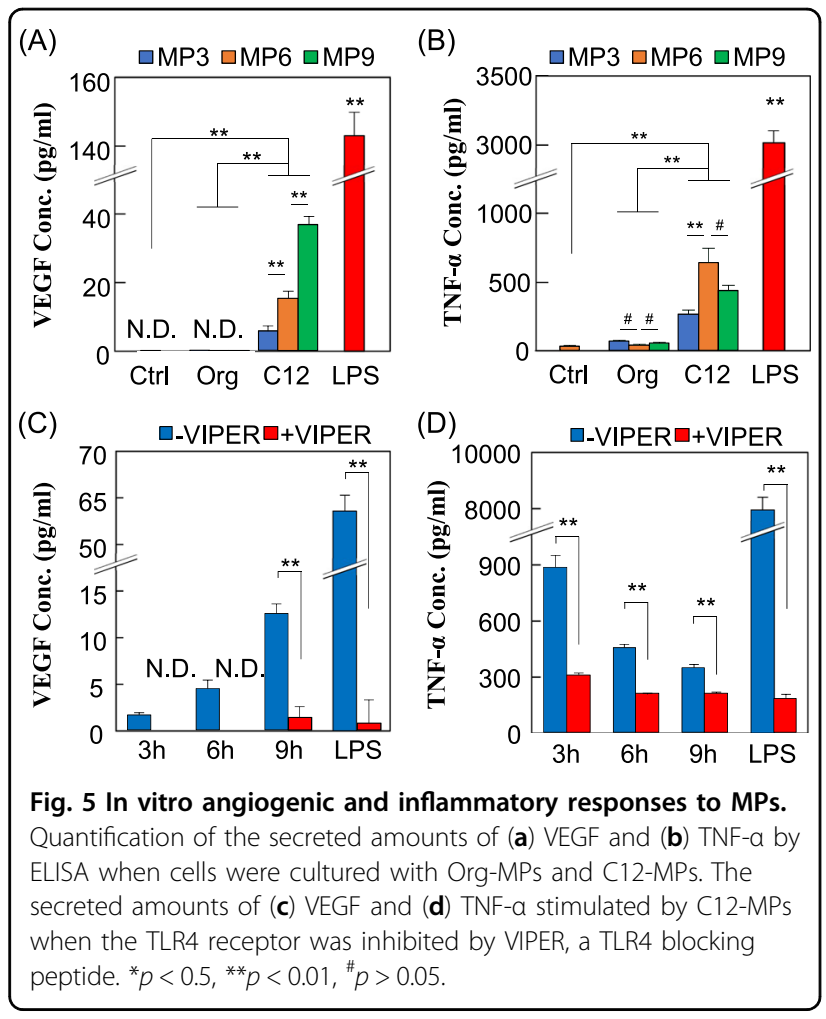

curves in each plot (Table 1). The Org-MP3 hydrogel had the shortest half-life in the collagenase solution among all the groups. The half-lives of the Org-MP6 and Org-MP9 hydrogels were $\sim 2 \mathrm{~h}$ longer than that of the Org-MP3 hydrogel; however, C12-MP hydrogels had half-lives that were two times longer, indicating that the C12-MP hydrogels were more stable in a physiological environment.

\section{Cellular response to the Org- and C12-MPs}

To evaluate the effects of the MPs on cell viability and angiogenic properties, MPs were cultured with RAW264 cells at low MP concentrations that did not aggregate. The average viabilities of RAW264 cells cultured with Org- and C12-MPs are shown in Fig. S5. Org-MP3, Org-MP6, and Org-MP9 showed enhanced cell viability compared with the tissue culture polystyrene (TCPS) control. Gelatin contains the Arg-Gly-Asp (RGD) sequence, which is recognized by cell integrins and promotes cell attachment and viability ${ }^{37,38}$. Therefore, RAW264 cells recognized the RGD sequences of the degraded Org-ApGltn molecules and the surface of Org-MP, which led to greater viability than the TCPS control. Although C12-MP3 at $0.05 \mathrm{w} / \mathrm{v} \%$ showed a similar effect as Org-MP3 on the viability, a higher concentration of C12-MP3 induced inhibitory effects on the cell viability. C12-MP6 and C12-MP9 at all concentrations had higher inhibitory effects on cell viability than C12-MP3 because the former MPs did not degrade during culture, and the hydrophobic moieties deposited on the C12-MP surface affected those effects. Moreover, C12MP6 and C12-MP9 are considered to be more frequently internalized in RAW264 cells because of their smaller size $^{39}$, which is attributed to the hydrophobic modification and longer crosslinking time ${ }^{40}$. Therefore, C12-MP6 and C12-MP9 increased the concentration of the hydrophobic moieties in the cells, which led to cytotoxicity.

The amounts of secreted VEGF and TNF- $\alpha$ from RAW264 cells cultured with Org- and C12-MPs were also evaluated. As shown in Fig. 5a, C12-MP3, C12-MP6, and C12-MP9 stimulated VEGF secretion at concentrations of $5.47,15.3$, and $36.8 \mathrm{pg} / \mathrm{ml}$, respectively, and a significant increase with increasing crosslinking time was confirmed. LPS at $10 \mathrm{ng} / \mathrm{ml}$ induced $143 \mu \mathrm{l}$ of VEGF secretion, which was more than 3.8 times greater than that induced by C12-MPs (Fig. 5a). The TCPS control and Org-MPs did not stimulate VEGF secretion from RAW264 cells. C12MPs also promoted the secretion of TNF- $\alpha$ compared with TCPS and Org-MPs (Fig. 5b). The longer crosslinking time of the C12-MPs tended to induce higher levels of VEGF and TNF- $\alpha$ expression because the longer crosslinking time of these MPs retained the particle shape and higher concentration of the dodecyl group on the surface of the C12-MPs during cell culture, leading to a greater interaction of the dodecyl group with cells. To evaluate the stimulation pathways of the enhanced secretion of VEGF and TNF- $\alpha$, RAW264 cells were pretreated with VIPER, the TLR4 inhibitor peptide, before the addition of C12-MPs and LPS. VIPER successfully suppressed the secretion of VEGF and TNF- $\alpha$ from RAW264 cells stimulated with C12-MPs and LPS (Fig. 5c, d). These results indicated that the C12-MP is a TLR4 ligand and stimulates downstream pathways, including the myeloid differentiation primary response protein (MyD) 88-TIR domain-containing adaptor protein (TIRAP)-dependent pathway ${ }^{10,41,42}$. NF-kB, the promoter of VEGF and the TNF- $\alpha$ gene ${ }^{42}$, was then activated, which enhances VEGF and TNF- $\alpha$ secretion.

\section{In vivo tissue responses to C12-MPs}

To evaluate the in vivo angiogenic properties of C12MPs, we employed hairless mice and observed the blood flow and histological responses. Figure 6a shows blood perfusion images of the PBS, Org-MP, and C12-MP hydrogels at the injection site taken with an LDPI system for up to 22 days. PBS and Org-MP hydrogels showed no increased blood flow for up to 22 days, whereas the C12MP3 hydrogel induced significantly higher blood perfusion on day 2 compared with not only PBS and Org-MPs but also the C12-MP6 and C12-MP9 hydrogels (Fig. 6b). Alternatively, the blood flow around the C12-MP3 hydrogels decreased by day 7 , returning to normal blood flow. The C12-MP6 hydrogel showed significantly higher blood flow from days 7 to 15 , followed by a decrease that 


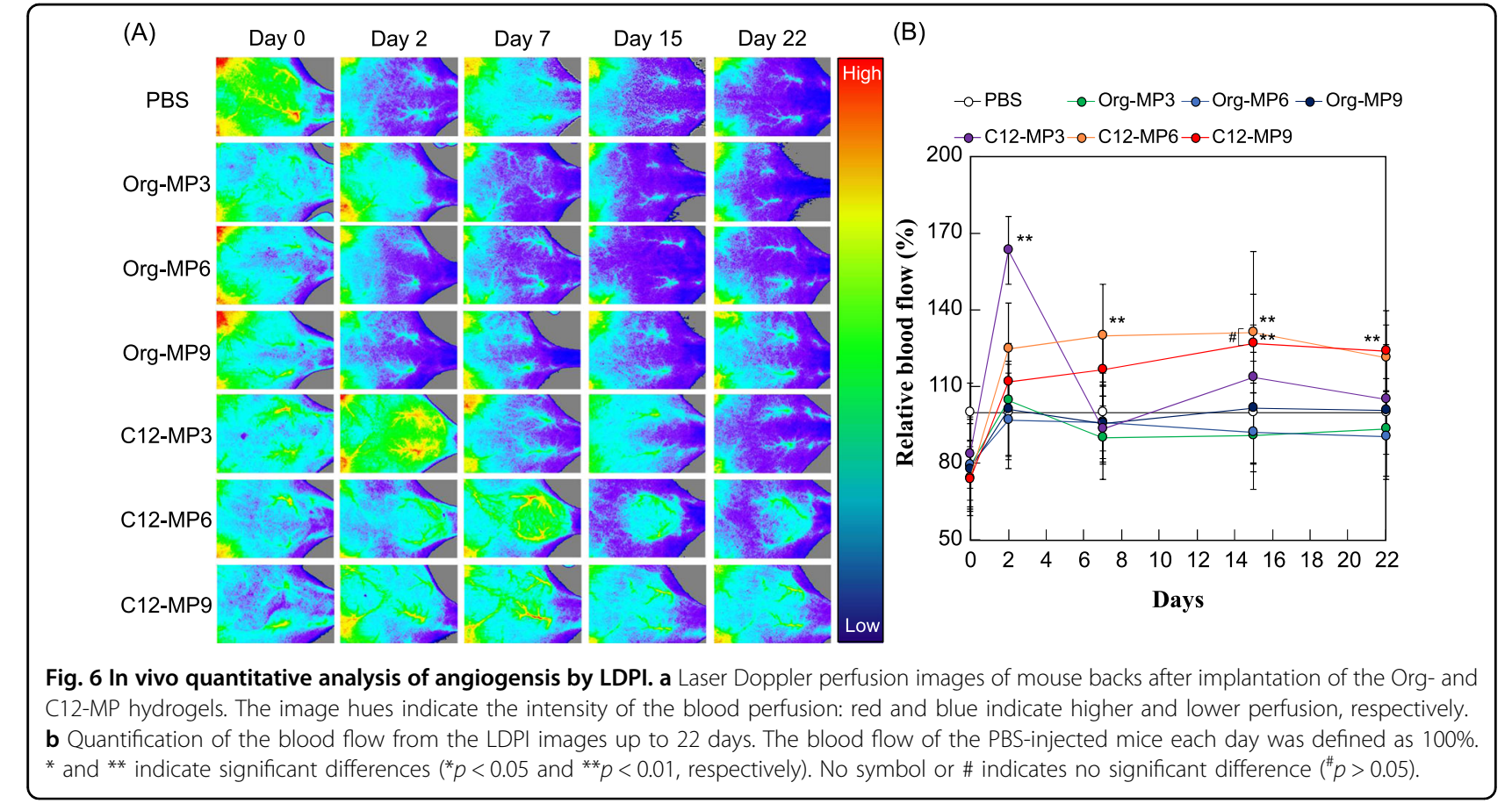

(B)

was not significant compared with PBS and Org-MP hydrogels at day 22 . On the other hand, the C12-MP9 hydrogel induced significantly higher blood flow from days 15 to 22 compared with all the other groups. The time course of the increased blood flow was dependent on the thermal crosslinking time. The C12-MP3 hydrogel was quickly degraded after injection, and the C12-ApGltn polymer increased around the injection site to promote angiogenesis, similar to our previous study ${ }^{17}$. Angiogenesis then decreased with enzymatic degradation and dispersion of the C12-MP3 hydrogel, and the blood flow also became equal to that of PBS and the Org-MP hydrogel injection. Alternatively, the C12-MP6 and C12-MP9 hydrogels maintained a high level of blood flow for up to 22 days due to the slow degradation rate, sustaining a high concentration of the C12-ApGltn molecule at the injection site. To evaluate the tissue responses on the C12-MP hydrogel, we also observed tissue sections of the Org-MP3 and C12-MP3 hydrogels on day 2 to quantify the degree of inflammation and angiogenesis (Fig. 7a). The stained area of NF-kB, the inflammation marker and promoter of the VEGF gene, increased to a greater extent after C12-MP3 hydrogel injection compared with PBS and Org-MP3 (Fig. 7b). Moreover, the stained area of CD31, an endothelial cell marker, was also significantly higher in the C12-MP3 hydrogel than in the PBS and Org-MP3 hydrogels, which indicates that the number of blood vessels increased around the C12-MP3 hydrogel injection site (Fig. 7a, c). Aoki et al. ${ }^{43}$ reported that poly(lactic-co-glycolic acid) (PLGA) nanosheets loaded with $250 \mathrm{ng}$ of fibroblast growth factor (bFGF) induced angiogenesis in vivo. The CD31-stained areas in tissue sections from the sham operation and unloaded PLGA nanosheets were comparable with our PBS and OrgMP3 hydrogel groups, respectively. However, the CD31stained area around the C12-MP3 hydrogel was $\sim 1.7$-fold greater than that of the bFGF-loaded PLGA nanosheet, indicating that the estimated efficacy of the C12-MP3 hydrogel was at least comparable to $250 \mathrm{ng}$ of bFGF. In addition, the red blood cells inside the blood vessels were confirmed from the tissue sections stained with $\mathrm{H} \& \mathrm{E}$ (Fig. 7a). The C12-MP3 hydrogel promoted angiogenesis around the injection site by inducing NF-кB. In addition, MPO staining revealed that the accumulation of granulocytes around the C12-MP3 hydrogel increased because of the enhanced secretion of inflammatory cytokine signaling through NF-kB (Fig. 7a). On the other hand, cell infiltration into Org- and C12-MP3 hydrogels was confirmed in MPOstained sections, indicating that Org- and C12-MP3 did not have severe cytotoxicity. Based on the in vitro experimental results that indicated that C12-ApGltn stimulated TLR4mediated VEGF secretion from RAW264 cells, C12-MP3 was considered to stimulate the TLR4-mediated pathway followed by increased secretion of NF-KB in vivo. Therefore, the proposed mechanisms of angiogenesis by the $\mathrm{C} 12$ MP hydrogel are: (1) binding of the C12-ApGltn molecule degraded from C12-MP on TLR4 in macrophages and activation of NF-kB; (2) enhanced secretion of VEGF by the activated NF- $\mathrm{KB}$ pathway; and (3) proliferation of endothelial cells by secreted VEGF, followed by blood perfusion (Fig. 8). Enhanced long-term angiogenesis could be beneficial for cell transplantation; however, angiogenesis based 


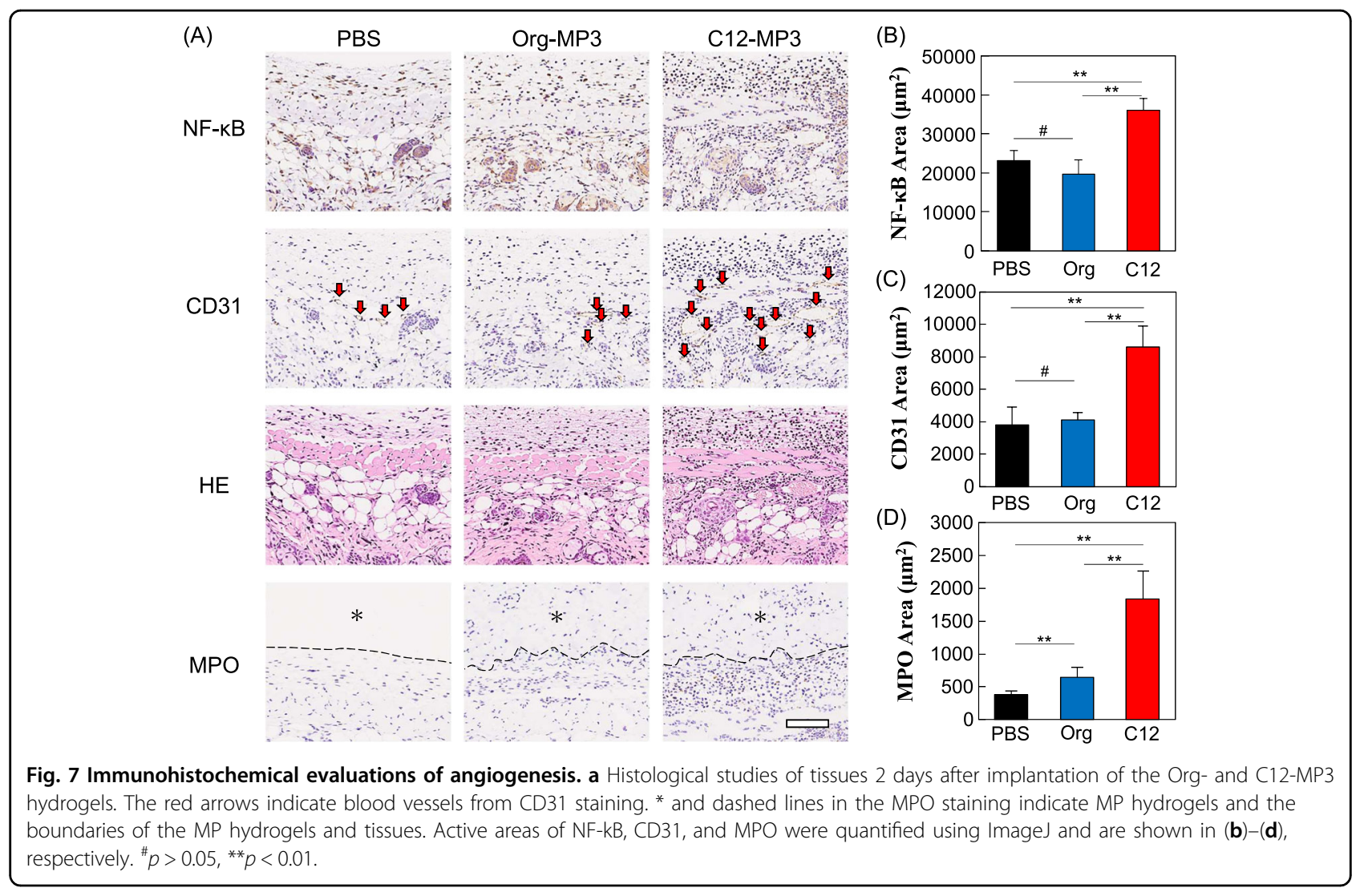

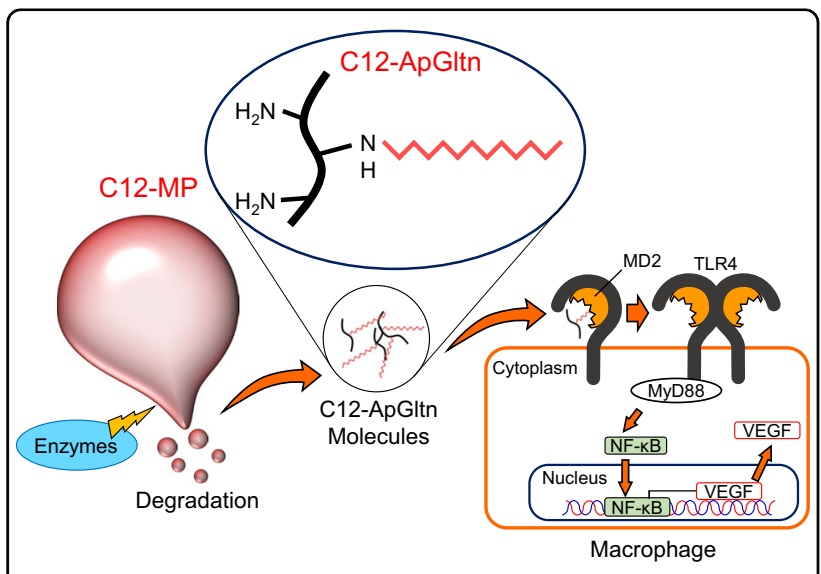

Fig. 8 Proposed mechanisms of angiogenesis by the C16-MP hydrogels.

on C12-MP hydrogels is accompanied by a weak inflammatory response and the secretion of inflammatory cytokines. Therefore, long-term secretion of inflammatory cytokines stimulated by C12-MP6 and C12-MP9 hydrogels may provoke the recruitment of fibroblasts and their proliferation, resulting in the formation of fibrous tissue near the injection site ${ }^{44}$. Hence, finding an optimal time point for cell transplantation by observing the time course of angiogenic and inflammatory responses stimulated by the C12-MP hydrogel is considered to be a clue for medical applications. Moreover, a combination of C12-MPs and anti-inflammatory drugs or materials to achieve a balance between inflammation and anti-inflammation may induce the recruitment of pericytes and the stabilization of newly formed blood vessels, leading to long-term stabilized angiogenesis ${ }^{45}$.

\section{Conclusion}

To achieve drug- and growth factor-free angiogenesis, we developed a C12-MP hydrogel that stimulates endogenous VEGF secretion and angiogenesis. C12-MPs stimulated the TLR4-mediated pathway and VEGF secretion in an in vitro study using the TLR4 blocking peptide. Moreover, the C12-MP hydrogel induced increased blood perfusion and the stained areas of NF- $\mathrm{KB}, \mathrm{CD} 31$, and MPO at the injection site. These results demonstrate that the C12-MP hydrogel is a potential material the construction of vascular beads to improve the survivability of implanted cells or tissues.

\section{Acknowledgements}

We are grateful to Dr. A. Nishiguchi, Mrs. M. Ishikawa, Mrs. M. Katano, Mrs. S. Watanabe, and Mrs. Y. Kurihara at the Polymeric Biomaterials Group, Polymers and Biomaterials Field, Research Center for Functional Materials, National Institute for Materials Science (NIMS) for their technical support. We would also 
like to thank Morphotechnology Co., Ltd. (Hokkaido, Japan) for making tissue sections. This work was financially supported in part by the Project for the Translational Research program: TR-SPRINT (Strategic Promotion for Practical Application of Innovative Medical Technology) from the Japan Agency of Medical Research and Development (Grant no. JP 20lm0203114h0001); "Innovation inspired by Nature" Research Support Program, SEKISUI CHEMICAL CO., LTD.; Uehara Memorial Foundation; and JSPS KAKENHI Grant-in-Aid for JSPS Fellows

(Grant no. 18J22422).

\section{Data availability}

The raw/processed data required to reproduce these findings cannot be shared at this time due to technical limitations.

\section{Conflict of interest}

The authors declare that they have no conflict of interest.

\section{Publisher's note}

Springer Nature remains neutral with regard to jurisdictional claims in published maps and institutional affiliations.

Supplementary information is available for this paper at https://doi.org/ 10.1038/s41427-020-0229-4.

Received: 8 March 2020 Revised: 18 April 2020 Accepted: 25 April 2020. Published online: 6 July 2020

\section{References}

1. Tabata, Y., Miyao, M., Ozeki, M. \& Ikada, Y. Controlled release of vascular endothelial growth factor by use of collagen hydrogels. J. Biomater. Sci. Polym. Ed. 11, 915-930 (2000).

2. Peattie, R. A. et al. Dual growth factor-induced angiogenesis in vivo using hyaluronan hydrogel implants. Biomaterials 27, 1868-1875 (2006).

3. Amsden, B. Novel biodegradable polymers for local growth factor delivery. Eur. J. Pharm. Biopharm. 97, 318-328 (2015).

4. Laham, R. J., Rezaee, M., Post, M., Xu, X. \& Sellke, F. W. Intrapericardial administration of basic fibroblast growth factor: myocardial and tissue distribution and comparison with intracoronary and intravenous administration. Catheter. Cardiovasc. Interv. 58, 375-381 (2003).

5. Lazarous, D. F. et al. Comparative effects of basic fibroblast growth factor and vascular endothelial growth factor on coronary collateral development and the arterial response to injury. Circulation 94, 1074-1082 (1996).

6. Ferrara, N., Gerber, H. P. \& LeCouter, J. The biology of VEGF and its receptors. Nat. Med. 9, 669-676 (2003).

7. Raetz, C. R. H. Biochemistry of endotoxins. Annu. Rev. Biochem. 59, 129-170 (1990).

8. Raetz, C. R. H. \& Whitfield, C. Lipopolysaccharide endotoxins. Annu. Rev. Biochem. 71, 635-700 (2002).

9. Alexander, C. \& Zähringer, U. Chemical structure of lipid A - The primary immunomodulatory center of bacterial lipopolysaccharides. Trends Glycosci. Glycotechnol. 14, 69-86 (2002).

10. Lee, J. Y. et al. Reciprocal modulation of toll-like receptor-4 signaling pathways involving MyD88 and phosphatidylinositol 3-kinase/AKT by saturated and polyunsaturated fatty acids. J. Biol. Chem. 278, 37041-37051 (2003).

11. Wong, S. W. et al. Fatty acids modulate toll-like receptor 4 activation through regulation of receptor dimerization and recruitment into lipid rafts in a reactive oxygen species-dependent manner. J. Biol. Chem. 284, 27384-27392 (2009).

12. Milanski, M. et al. Saturated fatty acids produce an inflammatory response predominantly through the activation of TLR4 signaling in hypothalamus: implications for the pathogenesis of obesity. J. Neurosci. 29, 359-370 (2009).

13. Huang, $\mathrm{S}$. et al. Saturated fatty acids activate TLR-mediated proinflammatory signaling pathways. J. Lipid Res. 53, 2002-2013 (2012).

14. Rocha, D. M., Caldas, A. P., Oliveira, L. L., Bressan, J. \& Hermsdorff, H. H. Saturated fatty acids trigger TLR4-mediated inflammatory response. Atherosclerosis $\mathbf{2 4 4}$ 211-215 (2016)
15. Wang, $Y$. et al. Saturated palmitic acid induces myocardial inflammatory injuries through direct binding to TLR4 accessory protein MD2. Nat. Commun. 8, 13997 (2017).

16. Yoshizawa, K., Mizuta, R. \& Taguchi, T. Enhanced angiogenesis of growth factor-free porous biodegradable adhesive made with hexanoyl groupmodified gelatin. Biomaterials 63, 14-23 (2015).

17. Mizuno, Y. \& Taguchi, T. Growth factor-free, angiogenic hydrogel based on hydrophobically modified Alaska pollock gelatin. J. Tissue Eng. Regen. Med. 13, 2291-2299 (2019).

18. Nishiguchi, A., Kurihara, Y. \& Taguchi, T. Underwater-adhesive microparticle dressing composed of hydrophobically-modified Alaska pollock gelatin for gastrointestinal tract wound healing. Acta Biomater. 99, 387-396 (2019).

19. Okuyama, T. \& Satake, K. On the preparation and properties of 2,4,6-trinitrophenyl-amino acids and -peptides. J. Biochem. 47, 454-466 (1960).

20. Habeeb, A. F. S. A. Determination of free amino groups in proteins by trinitrobenzenesulfonic acid. Anal. Biochem. 14, 328-336 (1966).

21. Adler-Nissen, J. Determination of the degree of hydrolysis of food protein hydrolysates by trinitrobenzenesulfonic acid. J. Agric. Food Chem. 27, 1256-1262 (1979).

22. Mizuno, Y., Mizuta, R., Hashizume, M. \& Taguchi, T. Enhanced sealing strength of a hydrophobically-modified Alaska pollock gelatin-based sealant. Biomater Sci. 5, 982-989 (2017)

23. Borch, R. F., Bernstein, M. D. \& Durst, H. D. The cyanohydridoborate anion as a selective reducing agent. J. Am. Chem. Soc. 93, 2897-2904 (1971).

24. Borch, R. F. \& Hassid, A. I. A new method for the methylation of amines. J. Org Chem. 37, 1673-1674 (1972).

25. Sato, S., Sakamoto, T., Miyazawa, E. \& Kikugawa, Y. One-pot reductive amination of aldehydes and ketones with a-picoline-borane in methanol, in water, and in neat conditions. Tetrahedron 60, 7899-7906 (2004).

26. Abdel-Magid, A. F., Carson, K. G., Harris, B. D., Maryanoff, C. A. \& Shah, R. D. Reductive amination of aldehydes and ketones with sodium triacetoxyborohydride. Studies on direct and indirect reductive amination procedures. J. Org. Chem. 61, 3849-3862 (1996).

27. Mizuno, Y. \& Taguchi, T. Promotion of cell migration into a hydrophobically modified Alaska pollock gelatin-based hydrogel. Macromol. Biosci. 19, 1900083 (2019).

28. Patra, S., Basak, P. \& Tibarewala, D. N. Synthesis of gelatin nano/submicron particles by binary nonsolvent aided coacervation (BNAC) method. Mater. Sci. Eng. C 59, 310-318 (2016).

29. Qin, Z., Joo, J., Gu, L. \& Sailor, M. J. Size control of porous silicon nanoparticles by electrochemical perforation etching. Part. Part. Syst. Charact. 31, 252-256 (2014).

30. Kozlov, N. K. et al. Recycling of silicon: from industrial waste to biocompatible nanoparticles for nanomedicine. Mater. Res. Express 4, 95026 (2017).

31. Zuidema, J. M., Rivet, C. J., Gilbert, R. J. \& Morrison, F. A. A protocol for rheological characterization of hydrogels for tissue engineering strategies. J. Biomed. Mater. Res. - Part B Appl. Biomater. 102, 1063-1073 (2014).

32. Browning, M. B., Cereceres, S. N., Luong, P. T. \& Cosgriff-Hernandez, E. M. Determination of the in vivo degradation mechanism of PEGDA hydrogels. J. Biomed. Mater. Res. - Part A 102, 4244-4251 (2014).

33. Lysakova-Devine, T. et al. Viral inhibitory peptide of TLR4, a peptide derived from vaccinia protein A46, specifically inhibits TLR4 by directly targeting MyD88 adaptor-like and TRIF-related adaptor molecule. J. Immunol. 185 4261-4271 (2010)

34. Bernert, D. B., Isenbügel, K. \& Ritter, H. Synthesis of a novel glycopeptide by polymeranalogous reaction of gelatin with mono-6-para-toluenesulfonyl$\beta$-cyclodextrin and its supramolecular properties. Macromol. Rapid Commun. 32, 397-403 (2011).

35. Rodin, V. V. \& Izmailova, V. N. NMR method in the study of the interfacial adsorption layer of gelatin. Colloids Surf. A Physicochem. Eng. Asp. 106, 95-102 (1996).

36. Nakamura, H. \& Ishii, M. Rheological behavior of concentrated monodispersed colloidal suspensions. Nihon Reoroji Gakkaishi 47, 1-7 (2019).

37. Gelse, K., Pöschl, E. \& Aigner, T. Collagens - Structure, function, and biosynthesis. Adv. Drug Deliv. Rev. 55, 1531-1546 (2003).

38. Davidenko, N. et al. Evaluation of cell binding to collagen and gelatin: a study of the effect of 2D and 3D architecture and surface chemistry. J. Mater. Sci. Mater. Med. 27, 148 (2016)

39. Nishiguchi, A. \& Taguchi, T. Designing an anti-inflammatory and tissueadhesive colloidal dressing for wound treatment. Colloids Surf. B Biointerfaces 188, 110737 (2020) 
40. Pacheco, P., White, D. \& Sulchek, T. Effects of microparticle size and Fc density on macrophage phagocytosis. PLOS ONE 8, e60989 (2013).

41. Itaya, $H$. et al. Expression of vascular endothelial growth factor in human monocyte/macrophages stimulated with lipopolysaccharide. Thromb. Haemost. 85, 171-176 (2001).

42. Kiriakidis, S. et al. VEGF expression in human macrophages is NF-kB-dependent: studies using adenoviruses expressing the endogenous NF-KB inhibitor $1 \mathrm{KBa}$ and a kinase-defective form of the IkB kinase 2. J. Cell Sci. 116, 665-674 (2003).
43. Aoki, S. et al. The efficacy of basic fibroblast growth factor-loaded poly(lacticco-glycolic acid) nanosheet for mouse wound healing. Wound Repair Regen. 25, 1008-1016 (2017).

44. Ueha, S., Shand, F. H. W. \& Matsushima, K. Cellular and molecular mechanisms of chronic inflammation-associated organ fibrosis. Front. Immunol. 3, 1-6 (2012).

45. Dulmovits, B. M. \& Herman, I. M. Microvascular remodeling and wound healing: a role for pericytes. Int. J. Biochem. Cell Biol. 44, 1800-1812 (2012). 